\title{
Pacific
}

Journal of

Mathematics

\section{EIGENVALUE COMPARISONS IN GRAPH THEORY}

GREGORY T. QUENELL 


\title{
EIGENVALUE COMPARISONS IN GRAPH THEORY
}

\author{
GREgory T. QUENELL
}

Let $\Gamma$ be a finite graph with degree bounded below by $k$. Let $\lambda_{1}, \lambda_{2}, \ldots, \lambda_{N}$ denote the eigenvalues of the adjacency operator on $\Gamma$, arranged in non-increasing order. We derive lower bounds for the first several $\lambda_{i}$ in terms of $k$ and the diameter of $\Gamma$.

Our bounds arise from a study of the roots of spherical eigenfunctions of the adjacency operator on a $k$-tree. We transplant these eigenfunctions onto $\Gamma$ to construct test functions whose Rayleigh quotients are easy to estimate.

\section{Introduction.}

A standard technique for estimating the eigenvalues of the Laplacian on a compact Riemannian manifold $M$ with bounded curvature is to pack the manifold with disjoint geodesic balls. The smallest Dirichlet eigenvalues of the Laplacian on the balls (which may be easier to estimate) can then be used as lower bounds for certain eigenvalues of the Laplacian on $M$. In this paper, we explore what happens when the same techniques are applied to the problem of estimating eigenvalues of the adjacency operator on finite graphs of bounded degree.

In Theorem 6.1, we show how eigenvalues of the adjacency operator on a finite graph $\Gamma$ may be bounded in terms of the biggest eigenvalues of the adjacency operator on "geodesic balls" in $\Gamma$. We find explicit bounds for the eigenvalues on the balls (Theorem 5.2), and in Theorem 6.2, we turn these into explicit estimates on certain eigenvalues of the adjacency operator on $\Gamma$.

\section{The setting: Linear algebra on Graphs.}

We begin by introducing the principal notions in the spectral theory of graphs and recalling a few results we will need from linear algebra.

Let $\Gamma$ be a connected, undirected graph with a finite number $n$ of verticès. We will denote the vertices $x_{1}, x_{2}, \ldots, x_{n}$. A function on $\Gamma$ is a map from the vertex set of $\Gamma$ to the real numbers. The set of functions on $\Gamma$ forms an 
$n$-dimensional vector space over $\mathbb{R}$. A canonical basis for this space is made up of the functions $\delta_{1}, \delta_{2}, \ldots, \delta_{n}$, where

$$
\delta_{i}\left(x_{j}\right)= \begin{cases}1 & \text { if } i=j \\ 0 & \text { otherwise. }\end{cases}
$$

There is also a canonical inner product on this function space. If $f$ and $g$ are functions on $\Gamma$, define

$$
\langle f, g\rangle=\sum_{i=1}^{n} f\left(x_{i}\right) g\left(x_{i}\right),
$$

and note that the canonical basis is orthonormal with respect to this inner product. We will denote the space of functions on $\Gamma$ (now a Hilbert space) as $L^{2}(\Gamma)$.

There is a natural self-adjoint linear operator on $L^{2}(\Gamma)$, called the adjacency operator, and denoted $A$. It is defined by

$$
(A f)(x)=\sum_{y \sim x} f(y),
$$

where $y \sim x$ means the vertex $y$ is joined to the vertex $x$ by an edge. The easy way to see that $A$ is indeed self-adjoint is to observe that its matrix with respect to the canonical basis is simply the adjacency matrix of $\Gamma$ which, since $\Gamma$ is undirected, must be symmetric.

The sequence of eigenvalues of $A$ forms the spectrum of $\Gamma$. The study of spectral graph theory involves looking for relations between the spectrum of a graph and its geometric properties. This is analogous to the field of spectral geometry on manifolds, where the spectrum of another self-adjoint operator, the Laplacian, is related to geometric features of a manifold. The analogy is very close, because any reasonable definition of a "Laplacian" on a graph (some operator that looks like heat diffusion) involves the adjacency operator in an important way. Consider, for example, a graph $\Gamma$ which is $k$ regular, meaning that every vertex in $\Gamma$ has degree $k$. A Laplacian operator $\Delta$ on such a graph, trying to look like minus the divergence of the gradient, might be defined as

$$
\begin{aligned}
\Delta f(x) & =\sum_{y \sim x}(f(x)-f(y)) \\
& =((k I-A) f)(x),
\end{aligned}
$$

where $I$ denotes the identity operator on $L^{2}(\Gamma)$.

A basic result in spectral geometry is a theorem of Cheng [4], giving upper bounds on the eigenvalues of the Laplacian on a compact Riemannian 
manifold $M$ in terms of the diameter of $M$ and curvature bounds on $M$. Intuitively, this result says that big manifolds have low fundamental frequencies. Our purpose here is to formulate the graph-theory analogue to Cheng's theorem, and derive lower bounds on the eigenvalues of the adjacency operator on a finite graph $\Gamma$ in terms of the diameter of $\Gamma$ and bounds on the degrees of its vertices. Our sample Laplacian in line (2) shows that the adjacency operator has the opposite sign from the usual Laplacian, so that lower bounds on eigenvalues of $A$ are the correct analogue to upper bounds on eigenvalues of $\Delta$.

If a graph $\Gamma$ is finite and $k$-regular, then the number $k$ is in the spectrum of $\Gamma$; the constant function is an eigenfunction for the value $k$. Furthermore, if $\Gamma$ is bipartite (meaning it contains no cycles of odd length) then the number $-k$ is also in the spectrum of $\Gamma$, corresponding to an eigenfunction with value 1 on half the vertices and -1 on the other half. A finite, $k$-regular graph is called Ramanujan if every eigenvalue $\lambda$ in its spectrum satisfies either $|\lambda|=k$ or $|\lambda| \leq 2 \sqrt{k-1}$.

Among other things, our results will explain the significance of the number $2 \sqrt{k-1}$ in this context, and clarify the assertion ([5]) that the secondgreatest eigenvalue of a finite, $k$-regular graph $\Gamma$ cannot be much smaller than $2 \sqrt{k-1}$ if $\Gamma$ is large.

We conclude this section by recalling two results from linear algebra: the variational characterization of eigenvalues and the Perron-Frobenius theorem.

Let $A$ be a self-adjoint linear operator on an $n$-dimensional Hilbert space $V$, so that $A$ has $n$ real eigenvalues $\lambda_{1} \geq \lambda_{2} \geq \cdots \geq \lambda_{n}$ with corresponding eigenvectors $v_{1}, v_{2}, \ldots, v_{n}$. Let $v$ be a non-zero vector in $V$. The Rayleigh quotient for $v$ is the quotient $\langle A v, v\rangle /\langle v, v\rangle$. Clearly, if $v$ happens to be an eigenvector $v_{i}$ of $A$, then the Rayleigh quotient for $v$ is equal to $\lambda_{i}$. In fact, each eigenvalue of $A$ is an extreme value of the Rayleigh quotient over an appropriate subspace of $V$. We will use this version of the variational characterization of eigenvalues:

Theorem (Rayleigh's Principle). With notation as above, for each index $1 \leq i \leq n$,

$$
\lambda_{i}=\max _{v \perp T_{i-1}} \frac{\langle A v, v\rangle}{\langle v, v\rangle}
$$

where $T_{i-1}$ is the subspace of $V$ spanned by the eigenvectors $v_{1}, v_{2}, \ldots, v_{i-1}$.

For a proof, see [9] or [6].

The Perron-Frobenius theorem is stated in terms of matrices rather than operators. 
A real matrix $M=\left(m_{i j}\right)$ is called non-negative if each of its entries is non-negative. A non-negative, square matrix $M$ is called irreducible if for each pair $(i, j)$, there is a non-negative integer $p$ such that the $(i, j)^{\text {th }}$ entry of $M^{p}$ is strictly positive. Since the $(i, j)^{\text {th }}$ entry of the $p^{\text {th }}$ power of the adjacency matrix of a graph $\Gamma$ is equal to the number of edge sequences of length $p$ connecting vertex $i$ to vertex $j$, it is clear that the adjacency matrix of a connected graph is irreducible.

For each $i$, the row sum $r_{i}$ of a matrix $M=\left(m_{i j}\right)$ is given by

$$
r_{i}=\sum_{j} m_{i j}
$$

Theorem (Perron-Frobenius). Let $M$ be a non-negative, square matrix, and suppose $M$ is irreducible. Let $r_{\min }$ and $r_{\max }$ be the minimum and maximum row sums of $M$, respectively. There is a unique eigenvector $v$ of $M$ all of whose entries are positive. The eigenvalue $\lambda$ corresponding to $v$ is the largest eigenvalue of $M$ and satisfies $r_{\min } \leq \lambda \leq r_{\max }$.

For a proof, see $[8]$.

\section{Trees and spherical functions.}

For each integer $k \geq 3$, let $\Gamma_{k}$ denote the $k$-tree, that is, the simplyconnected infinite graph each of whose vertices has degree $k$. The $k$-tree is the

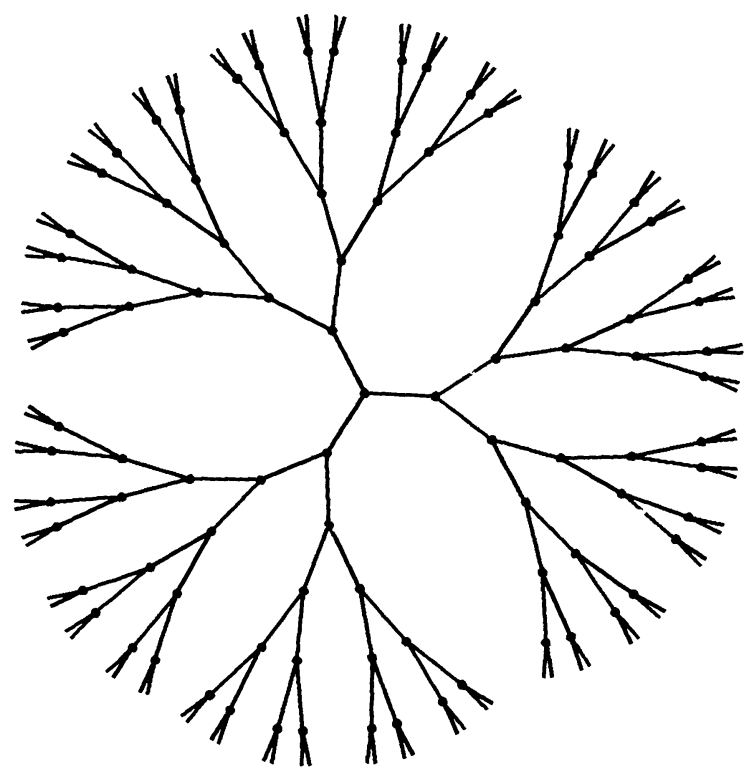

Figure 1. Part of the 3-tree. 
graph-theoretic analogue of a symmetric space. The number $k$ plays the role of negative curvature, in that the number of vertices in a ball of fixed radius in a $k$-tree (formal definitions to follow) increases with $k$, just as the volume of a unit ball in a symmetric space of negative curvature increases as the curvature gets "more negative."

Our reference point in spectral graph theory is the family of spherical eigenfunctions of the adjacency operator on $\Gamma_{k}$. These are analogous to the spherical eigenfunctions of the Laplacian on a symmetric space which one constructs using Bessel or Legendre functions (see [3]).

Given $\lambda \in(0,2 \sqrt{k-1})$ and $x_{0} \in \Gamma_{k}$, the spherical eigenfunction $S_{\lambda}$ on $\Gamma$ centered at $x_{0}$ is the function satisfying

(1) $A S_{\lambda}=\lambda S_{\lambda}$

(2) $S_{\lambda}\left(x_{0}\right)=1$

(3) $S_{\lambda}(x)$ depends only on the distance from $x$ to $x_{0}$.

We claim that there is a unique such function for each choice of $\lambda, x_{0}$, and $k \geq 3$, and will demonstrate this by constructing the function.

Since the value of $S_{\lambda}(x)$ depends only on the distance from $x$ to $x_{0}$, we can consider $S_{\lambda}$ as a function on the non-negative integers, and construct it as follows. Conditions (1) and (2) above imply that $S_{\lambda}$ satisfies the difference equation

$$
S_{\lambda}(r-1)+(k-1) S_{\lambda}(r+1)=\lambda S_{\lambda}(r)
$$

for $r \geq 1$, with initial conditions given by

$$
\begin{aligned}
S_{\lambda}(0) & =1 \\
k S_{\lambda}(1) & =\lambda .
\end{aligned}
$$

If we write

$$
S_{\lambda}(r)=c_{1} x_{1}^{r}+c_{2} x_{2}^{r},
$$

where $c_{1}$ and $c_{2}$ are coefficients to be determined later, then the difference equation (4) implies that $x_{1}$ and $x_{2}$ must satisfy the quadratic equation $1+(k-1) x^{2}=\lambda x$. Thus we have

$$
x_{1,2}=\frac{\lambda \pm \sqrt{\lambda^{2}-4(k-1)}}{2(k-1)} .
$$

Since we have assumed $0<\lambda<2 \sqrt{k-1}$, equation (8) implies that $x_{1}$ and $x_{2}$ are complex numbers with modulus $1 / \sqrt{k-1}$. This suggests writing

$$
x_{1,2}=\frac{1}{\sqrt{k-1}}\left(\frac{\lambda \pm \sqrt{\lambda^{2}-4(k-1)}}{2 \sqrt{k-1}}\right) .
$$


Let

$$
\theta=\arg \left(\frac{\lambda+\sqrt{\lambda^{2}-4(k-1)}}{2 \sqrt{k-1}}\right)
$$

so that $0<\theta<\pi / 2$. Then

$$
\begin{aligned}
S_{\lambda}(r) & =c_{1} x_{1}^{r}+c_{2} x_{2}^{r} \\
& =\frac{1}{\sqrt{k-1}^{r}}\left(c_{1} e^{i r \theta}+c_{2} e^{-i r \theta}\right) \\
& =\frac{1}{\sqrt{k-1}^{r}}\left(c_{3} \cos r \theta+c_{4} \sin r \theta\right) .
\end{aligned}
$$

Using the initial conditions $S_{\lambda}(0)=1$ and $S_{\lambda}(1)=\lambda / k$, we can determine that $c_{3}=1$ and $c_{4}=((k-2) / k) \cot \theta$. Thus our spherical eigenfunction on the $k$-tree is represented by the function

$$
S_{\lambda}(r)=\frac{1}{\sqrt{k-1}^{r}}\left(\cos r \theta+\frac{k-2}{k} \cot \theta \sin r \theta\right) .
$$

Reading $S_{\lambda}$ as a function of a continuous variable $r$, we observe that it has the form of a sine wave with period $2 \pi / \theta$ and exponentially decaying amplitude. If we rewrite $S_{\lambda}$ as

$$
S_{\lambda}(r)=\frac{c_{5}}{\sqrt{k-1}^{r}} \sin (r \theta+\alpha)
$$

equate this with our other expression for $S_{\lambda}$, and use the addition formula for sine, we can determine that the phase shift $\alpha$ is given by

$$
\tan \alpha=\frac{k}{k-2} \tan \theta
$$

with $0<\alpha<\pi / 2$, and that $c_{5}=\csc \alpha$.

Figure 2 shows some curves $y=S_{\lambda}(x)$ for $k=3$. The values of $\lambda$, given in the order in which the curves cross the $x$-axis, are $0.5,1,1.5,2$, and 2.5.

We summarize our investigation of spherical eigenfunctions in the following theorem.

Theorem 3.1. Let $k \geq 3$ be an integer and let $\lambda \in(0,2 \sqrt{k-1})$. Let

$$
\theta=\arg \left(\frac{\lambda+\sqrt{\lambda^{2}-4(k-1)}}{2 \sqrt{k-1}}\right) .
$$


Then the function

$$
S_{\lambda}(r)=\frac{\csc \alpha}{\sqrt{k-1}^{r}} \sin (r \theta+\alpha),
$$

where $\alpha=\arctan ((k /(k-2)) \tan \theta)$, is a spherical $\lambda$-eigenfunction of $A$ on $\Gamma_{k}$, in the sense that a function $\varphi$ on $\Gamma_{k}$ given by $\varphi(x)=S_{\lambda}\left(\operatorname{dist}\left(x, x_{0}\right)\right)$, for some fixed $x_{0}$, satisfies $A \varphi=\lambda \varphi$ and $S_{\lambda}\left(x_{0}\right)=1$.

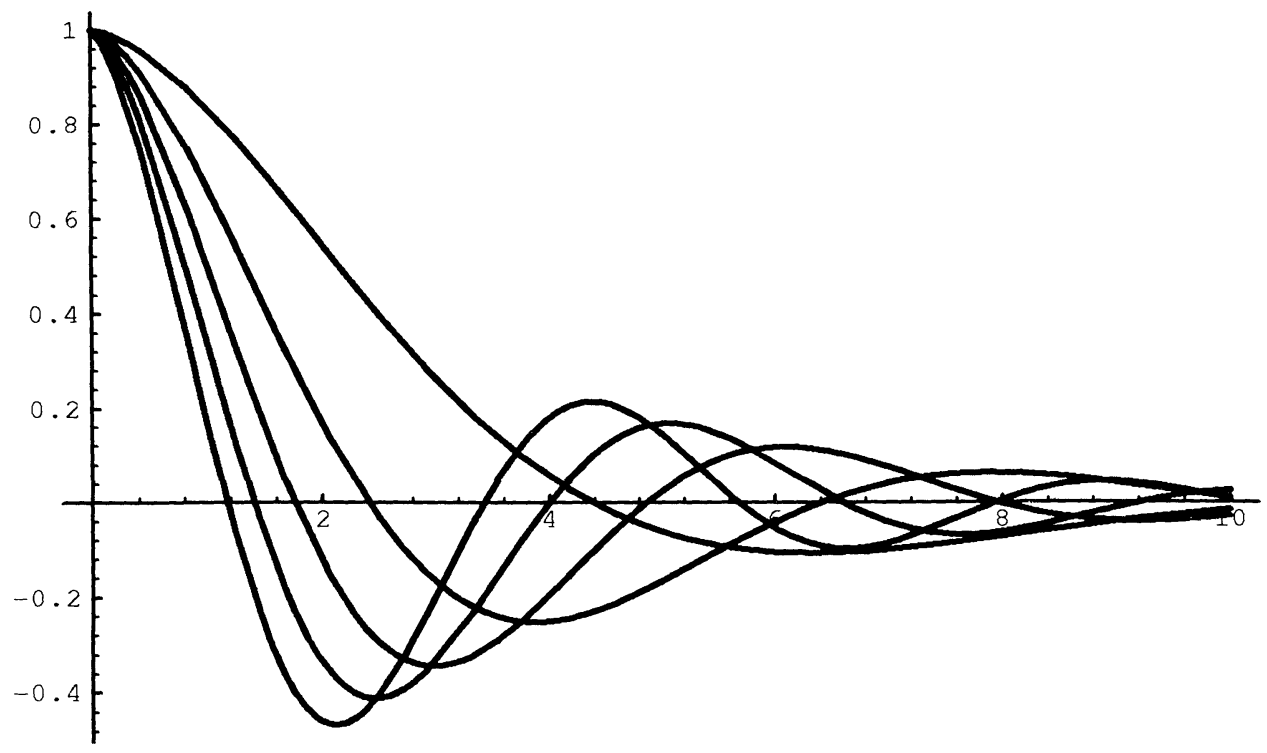

Figure 2. Graphs of some $S_{\lambda}$ functions.

We remark that the restriction $\lambda \in(0,2 \sqrt{k-1})$ is artificial. An eigenfunction $S_{\lambda}$ can be constructed for any $\lambda \in \mathbb{R}$ by making only minor changes to the development outlined above. See [2] or [7] for the details. In this paper, we will need only the functions $S_{\lambda}$ with $\lambda \in(0,2 \sqrt{k-1})$ and unless otherwise specified, any $\lambda$ we discuss will lie in this interval.

\section{Zeroes of spherical functions.}

Regarding $S_{\lambda}$ once again as a function of a continuous variable $r$, we now investigate where the first zero of $S_{\lambda}$ occurs. That is, we wish to identify the least positive $r$ such that $S_{\lambda}(r)=0$. Figure 2 suggests that the first positive root of $S_{\lambda}$ increases with $\lambda$. We establish this in the following theorem. 
Lemma 4.1. Let $S_{\lambda}$ denote the spherical eigenfunction with eigenvalue $\lambda$ on the $k$-tree. For each $r_{0}>1$, there is a real number $\lambda \in(0,2 \sqrt{k-1})$ such that $S_{\lambda}(r)>0$ for $0 \leq r<r_{0}$ and $S_{\lambda}\left(r_{0}\right)=0$.

Proof. We use the form of $S_{\lambda}$ given in Theorem 3.1, that is, $S_{\lambda}$ is equal to some positive exponential times the function $\sin (r \theta+\alpha)$. By the construction, we know $S_{\lambda}(0)=1$, so $\sin (0+\alpha)$ is strictly positive. Since $\theta$ and $\alpha$ are both positive, the first zero of $\sin (r \theta+\alpha)$ occurs when $r \theta+\alpha=\pi$. Solving for $r$, we find that the first zero of $S_{\lambda}$ occurs when

$$
r=\frac{\pi-\alpha}{\theta}
$$

From the definition of $\theta$ (Equation (10)), we get

$$
\cos \theta=\frac{\lambda}{2 \sqrt{k-1}}
$$

which shows that $\theta$ is a continuous, monotone decreasing function of $\lambda$ for $0<\lambda<2 \sqrt{k-1}$. As we noted earlier, the range of $\theta$ is the interval $(0, \pi / 2)$. The relation

$$
\tan \alpha=\frac{k}{k-2} \tan \theta
$$

(Equation (16)) shows that $\alpha$ is a continuous, monotone increasing function of $\theta$ for $0<\theta<\pi / 2$, and thus that $\alpha$ is a continuous, monotone decreasing function of $\lambda$.

Now the "first-zero" function $r=(\pi-\alpha) / \theta$ is a continuous, monotone increasing function of $\lambda$ on $0<\lambda<2 \sqrt{k-1}$. Furthermore, as $\lambda$ increases toward $2 \sqrt{k-1}$, both $\theta$ and $\alpha$ decrease to 0 , and the value of $r$ at the first zero increases without bound. As $\lambda$ decreases toward 0 , both $\theta$ and $\alpha$ increase to $\pi / 2$, and the value of $r$ at the first zero decreases toward 1. Since the range of the "first-zero" function is $(1, \infty)$ and the function is monotone, there is an inverse function giving, for each $r \in(1, \infty)$ the unique value of $\lambda$ between 0 and $2 \sqrt{k-1}$ such that $r$ is the first zero of $S_{\lambda}$.

We will use the notation $\Lambda(r)$ for the function constructed in Lemma 4.1. That is, for each $r>1$, the number $r$ will be the first zero of $S_{\Lambda(r)}$. In the next lemma, we derive an estimate on $\Lambda(r)$.

Lemma 4.2. For $r>1$,

$$
\Lambda(r)>2 \sqrt{k-1} \cos \left(\frac{\pi}{r+1}\right) .
$$


Proof. Let $\lambda=\Lambda(r)$, so that the first zero of $S_{\lambda}$ occurs at $r=(\pi-\alpha) / \theta$, where $\theta$ and $\alpha$ are the angles corresponding to the eigenvalue $\lambda$. Since $\theta$ and $\alpha$ both lie in $(0, \pi / 2)$ and $\tan \alpha=(k /(k-2)) \tan \theta$, we know $\tan \alpha>\tan \theta$, and thus that $\alpha>\theta$. This yields

$$
r=\frac{\pi-\alpha}{\theta}<\frac{\pi-\theta}{\theta}
$$

Solving for $\theta$ gives

$$
\theta<\frac{\pi}{r+1}
$$

Taking the cosine of both sides changes the direction of the inequality, giving

$$
\cos \theta>\cos \left(\frac{\pi}{r+1}\right) \text {. }
$$

Now $\cos \theta=\lambda /(2 \sqrt{k-1})$, so we have

$$
\frac{\lambda}{2 \sqrt{k-1}}>\cos \left(\frac{\pi}{r+1}\right)
$$

from which the result follows.

We will need one more result concerning the functions $S_{\lambda}$, which we state in the following lemma.

Lemma 4.3. Let $r_{0} \geq 1$ be an integer and let $\lambda=\Lambda\left(r_{0}\right)$. Then the function $S_{\lambda}$ is monotone decreasing on the integers $0,1, \ldots, r_{0}$.

Proof. We know that $S_{\lambda}$ is positive on the integers $0,1, \ldots, r_{0}-1$, that $S_{\lambda}(0)=1$, and that $S_{\lambda}$ satisfies the difference equation (4).

We proceed by induction, first noting that the difference equation for $S_{\lambda}$ implies that $S_{\lambda}(1)=\lambda / k$. Since $\lambda<2 \sqrt{k-1}$, we get

$$
S_{\lambda}(1)<\frac{2 \sqrt{k-1}}{k}
$$

For $k \geq 3$, the expression on the right is always less than 1 . Thus we have established the base case, that $S_{\lambda}(1)<S_{\lambda}(0)$.

Now assume that $1 \leq r \leq r_{0}-1$ and $S_{\lambda}(r)<S_{\lambda}(r-1)$. The difference equation for $S_{\lambda}$ implies that

$$
(k-1) S_{\lambda}(r+1)=\lambda S_{\lambda}(r)-S_{\lambda}(r-1) .
$$


Since $S_{\lambda}(r-1)>S_{\lambda}(r)$ and $\lambda<2 \sqrt{k-1}$, equation (26) gives the inequality

$$
\begin{aligned}
(k-1) S_{\lambda}(r+1) & <(\lambda-1) S_{\lambda}(r) \\
& <(2 \sqrt{k-1}-1) S_{\lambda}(r) \\
S_{\lambda}(r+1) & <\frac{2 \sqrt{k-1}-1}{k-1} S_{\lambda}(r) .
\end{aligned}
$$

For $k \geq 3$, the coefficient $(2 \sqrt{k-1}-1) /(k-1)$ is always less than 1 , and since $S_{\lambda}(r)$ is positive (because $r \leq r_{0}-1$ ), we have established that

$$
S_{\lambda}(r+1)<S_{\lambda}(r) \text {. }
$$

\section{Graph-theoretic balls.}

Next we examine the graph-theoretic analogues of geodesic balls in symmetric spaces and on manifolds.

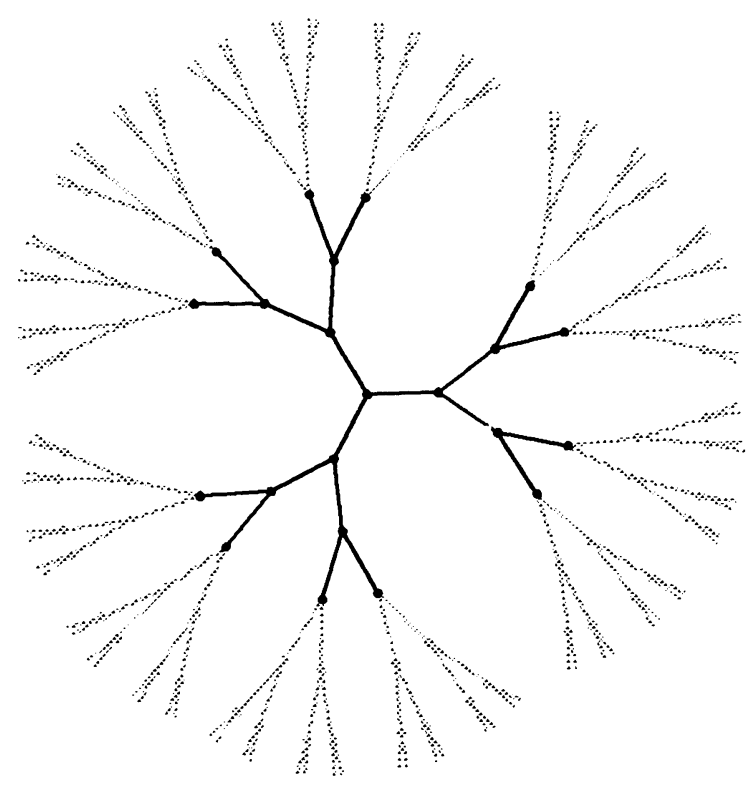

Figure 3. $V_{3}^{3}$ in 3 -tree.

We define the distance between two vertices $x$ and $y$ on a graph $\Gamma$ to be the number of edges traversed in the shortest path connecting $x$ to $y$. We 
denote the distance from $x$ to $y$ as $\operatorname{dist}(x, y)$. In a connected graph, $\operatorname{dist}(x, y)$ is always defined, and it is easy to check that it is a valid distance function.

Let $x_{0}$ be a vertex in a graph $\Gamma$, and let $n$ be a non-negative integer. The ball of radius $n$ about $x_{0}$, denoted $B\left(x_{0}, n\right)$, is the subgraph of $\Gamma$ induced by the set of vertices $x$ in $\Gamma$ with $\operatorname{dist}\left(x_{0}, x\right) \leq n$. (The subgraph induced by a set $S$ of the vertices of $\Gamma$ is a graph whose vertices are the elements of $S$ and whose edges are all the edges of $\Gamma$ which have both endpoints in $S$.)

Our reference graphs will be balls in the $k$-tree. Let $x_{0}$ be a vertex in $\Gamma_{k}$, and let $n$ be a non-negative integer. Because of the symmetry of $\Gamma_{k}$, the ball of radius $n$ around $x_{0}$ is isomorphic to the ball of radius $n$ around any other vertex in $\Gamma_{k}$. The shape of the ball does of course depend on $k$, and so we will use the notation $V_{n}^{k}$ to denote the ball of radius $n$ in $\Gamma_{k}$.

The quantity of interest to us is the largest eigenvalue of the adjacency operator on $V_{n}^{k}$, which we will denote $\lambda_{1}\left(V_{n}^{k}\right)$.

Theorem 5.1. For $n \geq 1$ and $k \geq 3$,

$$
\lambda_{1}\left(V_{n}^{k}\right)>2 \sqrt{k-1} \cos \left(\frac{\pi}{n+2}\right) .
$$

Proof. Let $x_{0}$ be the center of $V_{n}^{k}$, and define a test function $\varphi$ on $V_{n}^{k}$ by

$$
\varphi(x)=S_{\lambda}\left(\operatorname{dist}\left(x, x_{0}\right)\right),
$$

where $\lambda=\Lambda(n+1)$. We claim that $\varphi$ is an eigenfunction of the adjacency operator $A$ on $V_{n}^{k}$ with eigenvalue $\lambda$. To verify this, we examine $A \varphi(x)$ in three cases.

(1) $x=x_{0}$. Then $A \varphi(x)$ is a sum of $\varphi(y)$ over the $k$ vertices $y$ which are joined to $x_{0}$. The value of $\varphi$ at each such vertex is $S_{\lambda}(1)$, and we get $A \varphi(x)=k S_{\lambda}(1)=\lambda S_{\lambda}(0)=\lambda \varphi(x)$.

(2) $1 \leq r=\operatorname{dist}\left(x, x_{0}\right) \leq n-1$. The neighbors of $x$ include one vertex $y$ at distance $r-1$ from $x_{0}$ and $k-1$ vertices $z$ at distance $r+1$ from $x_{0}$. Thus

$$
\begin{aligned}
A \varphi(x) & =\varphi(y)+(k-1) \varphi(z) \\
& =S_{\lambda}(r-1)+(k-1) S_{\lambda}(r+1) \\
& =\lambda S_{\lambda}(r) \\
& =\lambda \varphi(x) .
\end{aligned}
$$

(3) $\operatorname{dist}\left(x, x_{0}\right)=n$. The vertex $x$ is joined to only one vertex $y$ in $V_{n}^{k}$, and $\operatorname{dist}\left(x_{0}, y\right)=n-1$. We also know that $S_{\lambda}(n+1)=0$, so we can write

$$
A \varphi(x)=\varphi(y)
$$




$$
\begin{aligned}
& =S_{\lambda}(n-1) \\
& =S_{\lambda}(n-1)+(k-1) S_{\lambda}(n+1) \\
& =\lambda S_{\lambda}(n) \\
& =\lambda \varphi(x) .
\end{aligned}
$$

Since $\lambda$ is an eigenvalue of the adjacency operator on $V_{n}^{k}$, we must have

$$
\lambda_{1}\left(V_{n}^{k}\right) \geq \lambda=\Lambda(n+1)>2 \sqrt{k-1} \cos \left(\frac{\pi}{n+2}\right) .
$$

The idea of using $S_{\lambda}$ to define a test function on a graph is called transplantation. This technique is very flexible, and can be applied to graphs other than $\Gamma_{k}$. In the next theorem, for example, we allow the graph $\Gamma$ to be multiply connected, and we even relax the $k$-regularity requirement.

A graph $\Gamma$ will be said to have minimal degree $k$ if every vertex of $\Gamma$ has degree greater than or equal to $k$.

Theorem 5.2. Let $\Gamma$ be a graph with minimal degree $k \geq 3$. Let $x_{0}$ be a vertex in $\Gamma$, let $n$ be a positive integer, and let $B=B\left(x_{0}, n\right)$ denote the ball around $x_{0}$ of radius $n$. Then the greatest eigenvalue of the adjacency operator on $B$, denoted $\lambda_{1}(B)$, satisfies

$$
\lambda_{1}(B)>2 \sqrt{k-1} \cos \left(\frac{\pi}{n+2}\right) .
$$

Proof. As in the proof of Theorem 5.1, we begin by defining a function $\varphi$ on $B$ by

$$
\varphi(x)=S_{\lambda}\left(\operatorname{dist}\left(x, x_{0}\right)\right)
$$

where $\lambda=\Lambda(n+1)$. Since distances on $\Gamma$ are well-defined, our function $\varphi$ is well-defined, and since all points in $B$ are within $n$ units of $x_{0}, \varphi$ is strictly positive on $B$.

We claim that the Rayleigh quotient for $\varphi,\langle A \varphi, \varphi\rangle /\langle\varphi, \varphi\rangle$, is greater than or equal to $\lambda$. Because the Rayleigh quotient for $\varphi$ is a lower bound for $\lambda_{1}(B)$, and $\lambda$ (by Theorem 4.2) is greater than $2 \sqrt{k-1} \cos (\pi /(n+2))$, we will be finished once we have established this claim.

The Rayleigh quotient for $\varphi$ is greater than or equal to $\lambda$ because $A \varphi$ is, pointwise, greater than or equal to $\lambda \varphi$. To see this, we examine $A \varphi(x)^{-}$in three cases.

(1) $x=x_{0}$. Then $A \varphi(x)$ is the sum of $\varphi(y)$ over all the vertices $y$ which are joined to $x_{0}$ by an edge. Since the degree of $x_{0}$ is at least $k$, this is 
a sum over at least $k$ vertices $y$, and the value of $\varphi$ at each such vertex is $S_{\lambda}(1)$. Since $S_{\lambda}(1)$ is positive, the sum is at least as large as $k S_{\lambda}(1)$, which is equal to $\lambda S_{\lambda}(0)$, which, in turn, is equal to $\lambda \varphi\left(x_{0}\right)$.

(2) $1 \leq r=\operatorname{dist}\left(x, x_{0}\right) \leq n-1$. Among the neighbors of $x$, there must be one vertex $y$ whose distance to $x_{0}$ is $r-1$. All the other neighbors $z$ of $x$ must lie within $r+1$ units of $x_{0}$, and there must be at least $k-1$ of them. Since $S_{\lambda}$ is decreasing, $\varphi$ must decrease with distance from $x_{0}$, and therefore the value of $\varphi$ at each $z$ is greater than or equal to $S_{\lambda}(r+1)$. This, along with the fact that $S_{\lambda}$ is positive in the relevant domain, gives us the following chain of inequalities.

$$
\begin{aligned}
A \varphi(x) & =\varphi(y)+\sum_{z} \varphi(z) \\
& \geq S_{\lambda}(r-1)+\sum_{z} S_{\lambda}(r+1) \\
& \geq S_{\lambda}(r-1)+(k-1) S_{\lambda}(r+1) \\
& =\lambda S_{\lambda}(r) \\
& =\lambda \varphi(x) .
\end{aligned}
$$

(3) $\operatorname{dist}\left(x, x_{0}\right)=n$. In this case, $x$ must have at least one neighbor $y$ whose distance to $x_{0}$ is $n-1$. Any other neighbors $z$ of $x$ satisfy $\varphi(z)>0$. Also, $S_{\lambda}(n+1)=0$, by our choice of $\lambda$. These observations justify the following chain of inequalities.

$$
\begin{aligned}
A \varphi(x) & =\varphi(y)+\sum_{z} \varphi(z) \\
& \geq \varphi(y) \\
& =S_{\lambda}(n-1)+(k-1) \cdot 0 \\
& =S_{\lambda}(n-1)+(k-1) S_{\lambda}(n+1) \\
& =\lambda S_{\lambda}(n) \\
& =\lambda \varphi(x) .
\end{aligned}
$$

We have established that $A \varphi(x) \geq \lambda \varphi(x)$ for each $x \in B$, and we know that $\varphi(x)>0$ for each $x \in B$, so

$$
\begin{aligned}
\langle A \varphi, \varphi\rangle & =\sum_{x \in B} A \varphi(x) \varphi(x) \\
& \geq \sum_{x \in B} \lambda \varphi(x) \varphi(x) \\
& =\lambda\langle\varphi, \varphi\rangle,
\end{aligned}
$$


showing that the Rayleigh quotient for $\varphi$ is indeed greater than $\lambda$.

We remark here that if $B$ itself has minimal degree $k_{1}$, then the PerronFrobenius theorem guarantees that $\lambda_{1}(B) \geq k_{1}$. However a ball $B$ in a graph $\Gamma$ with minimum degree $k$ can easily have vertices on its frontier with degree less than $k$. The balls $V_{n}^{k}$, for example, always contain vertices of degree 1 .

\section{Eigenvalue estimates.}

We will say that two balls $B_{1}$ and $B_{2}$ in a graph $\Gamma$ are edge-disjoint if the vertex set of $B_{1}$ is disjoint from that of $B_{2}$ and there is no edge in $\Gamma$ joining any vertex of $B_{1}$ to any vertex of $B_{2}$.

Theorem 6.1. Let $\Gamma$ be a finite graph. . Let $B_{1}, B_{2}, \ldots, B_{m}$ be balls in $\Gamma$ which are pairwise edge-disjoint. Then the $m^{\text {th }}$ largest eigenvalue of the adjacency operator on $\Gamma$, denoted $\lambda_{m}(\Gamma)$, satisfies

$$
\lambda_{m}(\Gamma) \geq \min _{i} \lambda_{1}\left(B_{i}\right),
$$

where $\lambda_{1}\left(B_{i}\right)$ denotes the largest eigenvalue of the adjacency operator on the subgraph $B_{i}$.

Proof. Let $\varphi_{1}, \ldots, \varphi_{m-1}$ be a set of eigenfunctions corresponding to the eigenvalues $\lambda_{1}, \ldots, \lambda_{m-1}$ of the adjacency operator $A$ on $\Gamma$. Because $A$ is selfadjoint, we know that the $\varphi_{i}$ can be chosen so that they are orthogonal, and thus they span an $(m-1)$-dimensional subspace $T_{m-1}$ of $L^{2}(\Gamma)$. The eigenvalue $\lambda_{m}$ is characterized by

$$
\lambda_{m}=\max _{\psi \perp T_{m-1}} \frac{\langle A \psi, \psi\rangle}{\langle\psi, \psi\rangle} .
$$

We will construct a test function from the eigenfunctions of the adjacency operators on all the balls $B_{i}$. For now, let $A_{i}$ denote the adjacency operator on the ball $B_{i}$. Let $\psi_{i}$ denote an eigenfunction corresponding to the first eigenvalue $\lambda_{1}\left(B_{i}\right)$ of the operator $A_{i}$ on each $B_{i}$. Extend each function $\psi_{i}$ to a function $\tilde{\psi}_{i}$ on all of $\Gamma$ by setting $\tilde{\psi}_{i}(x)=0$ for $x \notin B_{i}$. Because the balls $B_{i}$ are disjoint, the support of $\tilde{\psi}_{i}$ is disjoint from the support of $\tilde{\psi}_{j}$ if $i \neq j$, so the functions $\tilde{\psi}_{i}$ are all orthogonal to one another. The subspace of $L^{2}(\Gamma)$ spanned by the $\tilde{\psi}_{i}$ is therefore $m$-dimensional, and so it must contain a function which is orthogonal to $T_{m-1}$. That is, there are coefficients such that the function

$$
\tilde{\psi}=\sum_{i=1}^{m} \gamma_{i} \tilde{\psi}_{i}
$$


is orthogonal to every function in $T_{m-1}$. The Rayleigh quotient of $\tilde{\psi}_{i}$ will therefore be a lower bound for $\lambda_{m}(\Gamma)$.

To estimate this Rayleigh quotient, we observe first that for each $i$, the equality $\left\langle A \tilde{\psi}_{i}, \tilde{\psi}_{i}\right\rangle=\left\langle A_{i} \psi_{i}, \psi_{i}\right\rangle$ holds, where $A$ is the adjacency operator on all of $\Gamma$, and $A_{i}$ is the adjacency operator on just $B_{i}$. This fact, from which it follows that $\left\langle A \tilde{\psi}_{i}, \tilde{\psi}_{i}\right\rangle$ is actually equal to $\lambda_{1}\left(B_{i}\right)$, can be verified as follows.

$$
\begin{aligned}
\left\langle A \tilde{\psi}_{i}, \tilde{\psi}_{i}\right\rangle & =\sum_{x \in \Gamma} \sum_{\substack{y \sim x \\
y \in \Gamma}} \tilde{\psi}_{i}(y) \tilde{\psi}_{i}(x) \\
& =\sum_{x \in B_{i}} \sum_{\substack{y \sim x \\
y \in B_{i}}} \tilde{\psi}_{i}(y) \tilde{\psi}_{i}(x) \quad\left(\operatorname{supp}\left(\tilde{\psi}_{i}\right)=B_{i}\right) \\
& =\sum_{x \in B_{i}} \sum_{\substack{y \sim x \\
y \in B_{i}}} \psi_{i}(y) \psi_{i}(x) \\
& =\left\langle A \psi_{i}, \psi_{i}\right\rangle .
\end{aligned}
$$

We next make use of the fact that the $B_{i}$ are edge-disjoint to show that $\left\langle A \tilde{\psi}_{i}, \tilde{\psi}_{j}\right\rangle=0$ for $i \neq j$. We have

$$
\begin{aligned}
\left\langle A \tilde{\psi}_{i}, \tilde{\psi}_{j}\right\rangle & =\sum_{x \in \Gamma} \sum_{y \sim x} \tilde{\psi}_{i}(y) \tilde{\psi}_{j}(x) \\
& =\sum_{x \in B_{j}} \sum_{y \sim x} \tilde{\psi}_{i}(y) \tilde{\psi}_{j}(x) .
\end{aligned}
$$

But $B_{i}$ and $B_{j}$ are edge-disjoint, so $y \sim x$ and $x \in B_{j}$ imply that $y \notin B_{i}$, and thus that $\tilde{\psi}_{i}(y)=0$. Thus $\left\langle A \tilde{\psi}_{i}, \tilde{\psi}_{j}\right\rangle=0$.

And now we will compute the Rayleigh quotient for $\tilde{\psi}$ in two parts. First the numerator:

$$
\begin{aligned}
\langle A \tilde{\psi}, \tilde{\psi}\rangle & =\left\langle A \sum_{i} \gamma_{i} \tilde{\psi}_{i}, \sum_{j} \gamma_{j} \tilde{\psi}_{j}\right\rangle \\
& =\sum_{i, j} \gamma_{i} \gamma_{j}\left\langle A \tilde{\psi}_{i}, \tilde{\psi}_{j}\right\rangle \\
& =\sum_{i} \gamma_{i}^{2}\left\langle A \tilde{\psi}_{i}, \tilde{\psi}_{i}\right\rangle \\
& =\sum_{i} \lambda_{1}\left(B_{i}\right) \gamma_{i}^{2}\left\langle\tilde{\psi}_{i}, \tilde{\psi}_{i}\right\rangle .
\end{aligned}
$$

In computing the denominator, we will use the fact that the functions $\tilde{\psi}_{i}$ are mutually orthogonal. 


$$
\begin{aligned}
\langle\tilde{\psi}, \tilde{\psi}\rangle & =\left\langle\sum_{i} \gamma_{i} \tilde{\psi}_{i}, \sum_{j} \gamma_{j} \tilde{\psi}_{j}\right\rangle \\
& =\sum_{i, j} \gamma_{i} \gamma_{j}\left\langle\tilde{\psi}_{i}, \tilde{\psi}_{j}\right\rangle \\
& =\sum_{i} \gamma_{i}^{2}\left\langle\tilde{\psi}_{i}, \tilde{\psi}_{i}\right\rangle .
\end{aligned}
$$

For each $i$, let $\zeta_{i}=\gamma_{i}^{2}\left\langle\tilde{\psi}_{i}, \tilde{\psi}_{i}\right\rangle$, and let $Z=\sum \zeta_{i}$. Then the Rayleigh quotient for $\tilde{\psi}$ is equal to

$$
\sum_{i} \frac{\zeta_{i}}{Z} \lambda_{1}\left(B_{i}\right)
$$

Since the coefficients $\zeta_{i} / Z$ are all positive and add up to 1 , this Rayleigh quotient must be greater than $\min _{i} \lambda_{1}\left(B_{i}\right)$, and the proof is complete.

In the next theorem, we will bring into play our explicit estimates of the eigenvalues $\lambda_{1}\left(B_{i}\right)$, assuming bounded degree, to derive explicit lower bounds on $\lambda_{m}(\Gamma)$ for $m \geq 2$. For the moment, however, let us examine what Theorem 6.1 says about $\lambda_{1}(\Gamma)$. Since all the balls in the set $\left\{B_{1}\right\}$ are pairwise edge-disjoint (there are no pairs of them), $B_{1}$ may be taken to be any ball in $\Gamma$, and the theorem tells us that $\lambda_{1}(\Gamma) \geq \lambda_{1}\left(B_{1}\right)$, where $B_{1}$ is any ball in $\Gamma$. If the degree of $\Gamma$ is bounded below by $k$ and the radius of $B_{1}$ is $n$, Theorem 5.2 implies that $\lambda_{1}\left(B_{1}\right)>2 \sqrt{k-1} \cos (\pi /(n+2))$. We may take $n$ as large as we like (since $B_{1}$ will never intersect another ball, no matter what), so we can conclude that $\lambda_{1}(\Gamma) \geq 2 \sqrt{k-1}$. Unfortunately, this estimate is easily beaten by the Perron-Frobenius theorem, which says that if the degree of $\Gamma$ is bounded below by $k$, then $\lambda_{1}(\Gamma)$ is at least $k$. If, however, we can find a $B_{1}$ which is slightly smaller than $\Gamma$ and which has a minimal degree $k_{1}$ greater than $k$, then Theorem 6.1 applied with $B_{1}$ may yield a better estimate on $\lambda_{1}$ than Perron-Frobenius theorem does when applied to $\Gamma$ directly.

The diameter of a finite graph $\Gamma$ is the maximum of $\operatorname{dist}(x, y)$ over all pairs of vertices $x$ and $y$ in $\Gamma$. In the next theorem, we estimate the eigenvalues of the adjacency operator on $\Gamma$ in terms of the diameter of $\Gamma$.

Theorem 6.2. Let $\Gamma$ be a finite graph with diameter $d$ and minimal degree $k \geq 3$. Then for $2 \leq m \leq 1+d / 4$, the $m^{\text {th }}$ eigenvalue of the adjacency operator on $\Gamma$ satisfies

$$
\lambda_{m}(\Gamma)>2 \sqrt{k-1} \cos \left(\frac{\pi}{r+1}\right)
$$


where $r$ is the greatest integer less than or equal to $d /(2(m-1))$.

Proof. We claim there are $m$ vertices in $\Gamma$, separated pairwise by distances of at least $2 r$. If not, then the distance between any two vertices in $\Gamma$ less than $(m-1)(2 r) \leq d$, contradicting the fact that the diameter of $\Gamma$ is $d$. Choose $m$ such vertices $x_{1}, x_{2}, \ldots, x_{m}$, and for each one, let $B_{i}=B\left(x_{i}, r-1\right)$. The balls $B_{i}$ are edge-disjoint, because if $x \in B\left(x_{i}, r-1\right)$ and $y \in B\left(x_{j}, r-1\right)$ (with $i \neq j$ ) and $x$ and $y$ are joined by an edge, then the distance between $x_{i}$ and $x_{j}$ is less than or equal to $2(r-1)+1=2 r-1$, contrary to our choice of $x_{i}$ and $x_{j}$. Applying Theorem 6.1 , we conclude that $\lambda_{m}(\Gamma) \geq \min _{i} \lambda_{1}\left(B_{i}\right)$. Theorem 5.2 then tells us that each $\lambda_{1}\left(B_{i}\right)$ is greater than $2 \sqrt{k-1} \cos (\pi /(r+1))$, and the result follows.

One consequence of Theorem 6.2 for large (but finite) $k$-regular graphs may be seen as follows. The largest eigenvalue of $A$ on such a graph is $k$, and Theorem 6.2 gives the lower bound for $\lambda_{2}(\Gamma)$ as

$$
\lambda_{2}(\Gamma)>2 \sqrt{k-1} \cos \left(\frac{\pi}{r+1}\right)
$$

where $r$ is essentially $d / 2$. As $d$ gets large, the right side of (34) increases toward $2 \sqrt{k-1}$, so if $\Gamma_{1}, \Gamma_{2}, \ldots$ is a sequence of $k$-regular graphs such that

$$
\lim _{n \rightarrow \infty} \operatorname{diam}\left(\Gamma_{n}\right)=\infty
$$

we can conclude that

$$
\liminf _{n \rightarrow \infty} \lambda_{2}\left(\Gamma_{n}\right) \geq 2 \sqrt{k-1}
$$

Even for moderately large $k$-regular graphs, Theorem 6.2 does not allow $\lambda_{2}$ to be very far inside the interval $[-2 \sqrt{k-1}, 2 \sqrt{k-1}]$. The $k$-regular graphs which, in spite of this crowding effect, have $\lambda_{2}$ and all other eigenvalues except $\pm k$ in the given interval are honored with the name Ramanujan.

\section{Boundary case: radius zero.}

We can get an interesting application of Theorem 6.1 using balls of radius 0 . Mostly for notational convenience, all our previous theorems excluded this boundary case, but it is easy to state what happens when balls of radius zero are considered. Briefly, we will need to define $\Lambda(1)$ (it turns out to be 0 ), and so we will need to look at the function $S_{\lambda}$ when $\lambda=0$. Here are the boundary cases of our previous theorems, given in the order in which the theorems originally appeared. 
Theorem 7.1. (Compare with Theorem 3.1.) Let $k \geq 3$ be an integer. Then the function

$$
S_{0}(r)=\frac{1}{\sqrt{k-1}^{r}} \sin \left(\frac{r \pi}{2}+\frac{\pi}{2}\right)
$$

is a spherical 0-eigenfunction of $A$ on $\Gamma_{k}$.

Proof. This is just a matter of plugging $\lambda=0$ into the development of $S_{\lambda}$ in Section 3. The value of $\theta$ turns out to be $\pi / 2$, as does the value of $\alpha$.

Lemma 7.2. (Compare with Lemma 4.1.) $S_{0}(r)$ is the unique spherical eigenfunction on the $k$-tree which takes on the value 0 at $r=1$.

Proof. The difference equation defining $S_{\lambda}$ requires $\lambda S_{\lambda}(0)=k S_{\lambda}(1)$. Since $S_{\lambda}(1)=0$ and $S_{\lambda}(0)=1$, we conclude that $\lambda=0$.

Lemma 7.3. (Compare with Lemma 4.2.) $\Lambda(1)=0$.

Proof. Immediate.

A ball of radius 0 is a single vertex $x$. Since no other vertices are connected to $x$, the adjacency operator on a ball of radius 0 is the zero operator, and its only (and therefore largest) eigenvalue is 0 . Thus the boundary case of Theorem 5.1 is

Theorem 7.4. $\lambda_{1}\left(V_{0}^{k}\right)=0$.

Packing balls of radius 0 into a finite graph yields the following boundary case of Theorem 6.2 .

Theorem 7.5. Let $\Gamma$ be a finite graph with minimum degree $k \geq 3$ and diameter $d$. Let $n$ be the greatest integer less than or equal to $1+d / 2$. Then at least $n$ eigenvalues of the adjacency operator on $\Gamma$ are non-negative. Let $p$ be the greatest integer less than or equal to $1+d / 4$. Then at least $p$ eigenvalues of the adjacency operator on $\Gamma$ are positive.

Proof. $\Gamma$ must contain $n$ vertices separated from one another by distance at least 2 . If not, then the diameter of $\Gamma$ is less than $2(n-1)$, and therefore less than $d$, contrary to hypothesis. We consider these $n$ vertices as $n$ balls of radius 0 , and apply Theorems 6.1 and 7.4 to conclude that $\lambda_{n}(\Gamma) \geq 0$. The second assertion is immediate from Theorem 6.2.

\section{References}

[1] P. Bérard, Spectral geometry: direct and inverse problems, Lecture notes in mathematics, 1207, Springer Verlag, Berlin, 1986. 
[2] R. Brooks, The spectral geometry of k-regular graphs, Journal d'Analyse, 57 (1991), 120-151.

[3] I. Chavel, Eigenvalues in Riemannian Geometry, Academic Press, Inc., Orlando, FL, 1984.

[4] S.-Y. Cheng, Eigenvalue comparison theorems and its geometric applications, Math. Z., 143 (1975), 289-297.

[5] A. Lubotzky, R. Phillips and P. Sarnak, Ramanujan graphs, Combinatorica, 8(3) (1988), 261-277.

[6] B. Noble and J.W. Daniel, Applied Linear Algebra, Prentice-Hall, Englewood Cliffs, NJ, 1977.

[7] G. Quenell, Spectral diameter estimates for $k$-regular graphs, Advances in mathematics, 106(1) (1994), 122-148.

[8] E. Seneta, Non-negative matrices and Markov chains, Springer-Verlag, New York, 1981.

[9] H.F. Weinberger, Variational Methods for Eigenvalue Approximation, Regional Conference Series in Applied Mathematics, 15, Society for Industrial and Applied Mathematics, Philadelphia, 1974.

Received August 22, 1994.

Oberlin College

OBERLIN, OH 44074

E-mail address: quenell@cs.oberlin.edu 



\title{
PACIFIC JOURNAL OF MATHEMATICS
}

\author{
Founded in 1951 by
}

\author{
$\begin{array}{ll}\text { E. F. Beckenbach (1906-1982) } & \text { F. Wolf (1904-1989) }\end{array}$
}

\section{EDITORS}

Sun-Yung A. Chang (Managing Editor) Robert Finn University of California

Los Angeles, CA 90095-1555

pacific@math.ucla.edu

\section{F. Michael Christ}

University of California

Los Angeles, CA 90095-1555

christ@math.ucla.edu

Nicholas Ercolani

University of Arizona

Tucson, AZ 85721

ercolani@math.arizona.edu
Stanford University

Stanford, CA 94305

finn@gauss.stanford.edu

Steven Kerckhoff

Stanford University

Stanford, CA 94305

spk@gauss.stanford.edu

Martin Scharlemann

University of California

Santa Barbara, CA 93106

mgscharl@math.ucsb.edu

\section{Gang Tian}

Massachusettes Institute of Technology

Cambridge, MA 02139

tian@math.mit.edu

\section{S. Varadarajan University of California Los Angeles, CA 90095-1555 vsv@math.ucla.edu \\ Dan Voiculescu \\ University of California \\ Berkeley, CA 94720 \\ dvv@math.berkeley.edu}

\section{SUPPORTING INSTITUTIONS}

ACADEMIA SINICA, TAIPEI

CALIF. INST. OF TECHNOLOGY

CHINESE UNIV. OF HONG KONG

HONG KONG UNIV. OF SCI. \& TECH.

KEIO UNIVERSITY

MACQUARIE UNIVERSITY

MATH. SCI. RESEARCH INSTITUTE

NEW MEXICO STATE UNIV.

OREGON STATE UNIV.

PEKING UNIVERSITY

RITSUMEIKAN UNIVERSITY

STANFORD UNIVERSITY

\author{
TOKYO INSTITUTE OF TECHNOLOGY \\ UNIVERSIDAD DE LOS ANDES \\ UNIV. OF ARIZONA \\ UNIV. OF BRITISH COLUMBIA \\ UNIV. OF CALIF., BERKELEY \\ UNIV. OF CALIF., DAVIS \\ UNIV. OF CALIF., IRVINE \\ UNIV. OF CALIF., LOS ANGELES \\ UNIV. OF CALIF., RIVERSIDE \\ UNIV. OF CALIF., SAN DIEGO \\ UNIV. OF CALIF., SANTA BARBARA
}

UNIV. OF CALIF., SANTA CRUZ

UNIV. OF HAWAII

UNIV. OF MELBOURNE

UNIV. OF MONTANA

UNIV. NACIONAL AUTONOMA DE MEXICO

UNIV. OF NEVADA, RENO

UNIV. OF OREGON

UNIV. OF SOUTHERN CALIFORNIA

UNIV. OF UTAH

UNIV. OF WASHINGTON

WASHINGTON STATE UNIVERSITY

The supporting Institutions listed above contribute to the cost of publication of this Journal, but they are not owners or publishers and have no responsibility for its contents or policies.

Manuscripts must be prepared in accordance with the instructions provided on the inside back cover.

The table of contents and the abstracts of the papers in the current issue, as well as other information about the Pacific Journal of Mathematics, may be found on the Internet at http://www.math.uci.edu/pjm.html.

The Pacific Journal of Mathematics (ISSN 0030-8730) is published monthly except for July and August. Regular subscription rate: $\$ 245.00$ a year (10 issues). Special rate: $\$ 123.00$ a year to individual members of supporting institutions.

Subscriptions, back issues published within the last three years and changes of subscribers address should be sent to Pacific Journal of Mathematics, P.O. Box 4163, Berkeley, CA 94704-0163, U.S.A. Prior back issues are obtainable from Kraus Periodicals Co., Route 100, Millwood, NY 10546.

The Pacific Journal of Mathematics at the University of California, c/o Department of Mathematics, 981 Evans Hall, Berkeley, CA 94720 (ISSN 0030-8730) is published monthly except for July and August. Second-class postage paid at Berkeley, CA 94704, and additional mailing offices. POSTMASTER: send address changes to Pacific Journal of Mathematics, P.O. Box 6143, Berkeley, CA 94704-0163.

\section{PUBLISHED BY PACIFIC JOURNAL OF MATHEMATICS at University of California,} Berkeley, CA 94720, A NON-PROFIT CORPORATION

This publication was typeset using AMS-LATEX,

the American Mathematical Society's TEX macro system.

Copyright (C) 1995 by Pacific Journal of Mathematics 


\section{PACIFIC JOURNAL OF MATHEMATICS}

\section{Volume $176 \quad$ No. $2 \quad$ December 1996}

One remark on polynomials in two variables

ENRIQUe ARTAl BARTOLO and PIERRETTE CASSOU-NOGUÈS

Divergence of the normalization for real Lagrangian surfaces near complex tangents XIANGHONG GONG

Classification of the stable homotopy types of stunted lens spaces for an odd prime JESUS GONZALEZ

Plancherel formulae for non-symmetric polar homogeneous spaces

JING-SONG HUANG

A uniqueness theorem for the minimal surface equation

JENN-FANG HWANG

Differential Galois groups of confluent generalized hypergeometric equations: an approach

using Stokes multipliers

Claudine Mitschi

Oscillatory theorem and pendent liquid drops

KIMIAKI NARUKAWA and TAKASHI SUZUKI

Local and global plurisubharmonic defining functions

ALAN NOELL

Specializations and a local homeomorphism theorem for real Riemann surfaces of rings

M. J. DE LA PUENTE

Eigenvalue comparisons in graph theory

GREGORY T. QUENELL

Applications of loop groups and standard modules to Jacobians and theta functions of isospectral curves

WILLI SCHWARZ

Bridged extremal distance and maximal capacity

ROBERT E. THURMAN

Imbedding and multiplier theorems for discrete Littlewood-Paley spaces

IGOR E. VERBITSKY

On constrained extrema

THOMAS VOGEL

Heat flow of equivariant harmonic maps from $\mathbb{B}^{3}$ into $\mathbb{C P} \mathbb{P}^{2}$

YUANLONG XIN

Proof of Longuerre's theorem and its extensions by the method of polar coordinates

ZHIHONG YU

Correction to: "Special generating sets of purely inseparable extension fields of unbounded exponent"

BONIFACE IHEMOTUONYE EKE 